\section{Band 16, Heft 3, September 2006}

\section{Editorial}

164 Gruppentherapie

Linden, M. (Teltow/Berlin)

Originalarbeiten

166 Sexuelle Dysfunktionen bei Patienten einer verhaltenstherapeutischen Hochschulambulanz:

Häufigkeit, Erkennen, Behandlung

Reinecke, A.; Schöps, D.; Hoyer, J. (Dresden)

173 Ambulante verhaltenstherapeutische

Gruppentherapie bei Zwangsstörungen -

ein effektiver Behandlungsansatz?

Meier, S.; Fricke, S.; Moritz, S.; Hand, I. (Hamburg); Rufer, M. (Zürich)

184 Ein kognitiv-verhaltenstherapeutisches Gruppentraining für Frauen mit Wechseljahresbeschwerden - Ergebnisse einer kontrollierten Gruppenstudie

von Bültzingslöwen, K.; Pfeifer, M.; Kröner-Herwig, B. (Göttingen)

193 Genetische Aspekte in der Adipositas-Beratung Auswirkungen auf Körperakzeptanz und subjektives Wohlbefinden

Dierk, J.-M.; Conradt, M.; Schlumberger, P.; Rauh, E.; Albohn, C.; Hinney, A.; Hebebrand, J.; Rief, W. (Marburg)

\section{Übersichtsarbeit}

201 Effekte und Kosten universaler Prävention von Internalisierungsstörungen bei Kindern und Jugendlichen

Pössel, P. (Nashville, TN); Schneider, S. (Basel); Seemann, S. (Tübingen)

\section{Für die Praxis}

212 Gruppentherapiemodul zur Stärkung des Selbstwertes bei Patientinnen mit BorderlinePersönlichkeitsstörung

Jacob, G.A.; Richter, H. (Freiburg i.Br.); Lammers, C.-H. (Berlin); Bohus, M. (Mannheim); Lieb, K. (Freiburg i.Br.)
Vol. 16, Issue 3, September 2006

Editorial

164 Group Therapy

Linden, M. (Teltow/Berlin)

Original Articles

166 Sexual Dysfunctions in Patients of a CBT Outpatient University Clinic: Frequency,

Recognition, and Treatment

Reinecke, A.; Schöps, D.; Hoyer, J. (Dresden)

173 Outpatient Behavioural Group Therapy for Obsessive Compulsive Disorder - an Effective Treatment Approach?

Meier, S.; Fricke, S.; Moritz, S.; Hand, I. (Hamburg); Rufer, M. (Zürich)

184 A Cognitive-Behavioral Group Intervention for Menopausal Women - Results of a Randomized Controlled Study

von Bültzingslöwen, K.; Pfeifer, M.; Kröner-Herwig, B. (Göttingen)

193 Genetic Aspects in Obesity Counselling - Effects on Body Acceptance and Subjective Well-Being Dierk, J.-M.; Conradt, M.; Schlumberger, P.; Rauh, E.; Albohn, C.; Hinney, A.; Hebebrand, J.; Rief, W. (Marburg)

Review Article

201 Effects and Costs of Universal Prevention of Internalization Disorders in Children and Adolescents Pössel, P. (Nashville, TN); Schneider, S. (Basel); Seemann, S. (Tübingen)

For the Practitioner

212 Group Therapy Module to Enhance Self-Esteem in Patients with Borderline Personality Disorder Jacob, G.A.; Richter, H. (Freiburg i.Br.); Lammers, C.-H. (Berlin); Bohus, M. (Mannheim); Lieb, K. (Freiburg i.Br.)

\section{KARGER}

Fax +49761 4520714

E-mail Information@Karger.de

www.karger.com

\section{(c) 2006 S. Karger GmbH, Freiburg}

Artikel (Volltext) und Inhaltsverzeichnisse

sowie das vorläufige Inhaltsverzeichnis des nächsten Heftes: www.karger.com/ver_bk.htm 


\section{Verhaltenstherapie}

\section{Band 16, Heft 3, September 2006}

Interview

222 Ulrike Worringen: «Patientenschulung ist ein zentraler Behandlungsbaustein in der medizinischen Rehabilitation» Linden, M. (Berlin)

225 Neuigkeiten vom Buch- und Zeitschriftenmarkt

230 Fort- und Weiterbildung

234 Mitteilungen der Verbände

238 Tagungen und Kongresse

237 Impressum

U3 Hinweise für Autoren (3. Umschlagseite)

U2 Erläuterungen zum Titelbild (2. Umschlagseite) Leibl, C.; Vogt, B.; Jaschke, C. (Prien/Chiemsee)

Einen Ausblick auf den Inhalt der kommenden Hefte finden Sie auf Seite 240.
Vol. 16, Issue 3, September 2006

Interview

222 Ulrike Worringen: 'Patient Education Is a Core Element of Medical Rehabiliation' Linden, M. (Berlin)

225 Articles and Books

230 Education

234 Information by Behavior Therapy Associations

238 Meetings and Conferences

237 Imprint

C3 Guidelines for Authors (Inside back cover)

C2 Explanations on the Title (Inside front cover) Leibl, C.; Vogt, B.; Jaschke, C. (Prien/Chiemsee)

Forthcoming papers are listed on page 240.

\section{KARGER}

Fax +497614520714

E-mail Information@Karger.de

www.karger.com (c) 2006 S. Karger GmbH, Freiburg

Artikel (Volltext) und Inhaltsverzeichnisse

sowie das vorläufige Inhaltsverzeichnis des nächsten Heftes: www.karger.com/ver_bk.htm 